\title{
Study on the Application of GIS in the Same Frequency and Phase AC Voltage Test Method
}

\author{
Gu Qiang, Weng Xiaoyu, Yuan Ting, Wang Liang \\ Panjin Power Supply Company, Liaoning Electric Power Company Limited, State Grid, China, \\ 16295036@qq.com
}

Keywords: GIS under the same frequency and phase, AC voltage withstand test, method application.

\begin{abstract}
In order to carry out comprehensive analysis and study on the application of GIS in the same frequency and phase AC voltage withstand test method,this paper studied the same frequency and phase AC voltage withstand test of GIS, briefly described the test device and the connection principle of same frequency and phase AC GIS voltage test, and analyzed the voltage variation of GIS device bus isolation switch port in detail.Hope to provide reference and help for the use of GIS equipment in $\mathrm{AC}$ voltage test.
\end{abstract}

The traditional GIS AC voltage withstand test requires the whole performance of the power failure of adjacent to the test part and the operation part to finish it successfully.However, the same frequency and phase AC withstand voltage test of GIS refers to the same frequency and phase AC withstand voltage test of expansion or maintenance of the interval part of GIS equipment under the condition of keeping the adjacent test section and running part without power outage. Compared with the traditional test method, the test method of GIS with the same frequency and phase has some significant and outstanding advantages.

\section{Overview of test device and principle of GIS AC voltage withstand test with the same frequency and phase}

That the equipment will be used mainly in the test process contain the test power supply with the same frequency and phase, voltage measuring device, protective resistor, controller, transformer and reactor and so on.

Firstly, the voltage signal is obtained from the running bus of PT, which is used as the reference signal of the same frequency and phase AC voltage test of GIS.Then, use PLL and linear push-pull amplifier technology to control the final output voltage of the test device in connection in the circuit,to keep its frequency and phase the same with the voltage of the operation bus.

after a series of matching and filtering, the input signal of the phase locked loop would extract the reference signal of voltage phase effectively, and send it to the comparator of the voltage phase.Try to control the voltage phase in the numerical consistency on the same or are very close to the similarity range by adjusting the phase difference between the frequency of the sampled signal and PT secondary signal constantly.Then the GIS AC voltage withstand test system based on the same frequency and phase voltage is successfully realized. If the voltage phase difference of the test system is found and detected more than the overall protection value set by the user, the test voltage on the GIS isolation switch will sent out and the signal that the system was broken and make emergency separation treatment immediately,and stop outputting the abnormal voltage to test system and abnormal output of frequency conversion power.

\section{Analysis on the break voltage of isolation switch in the test process of GIS equipment bus}

According to the terms of the provisions of the relevant rules and regulations,all GIS equipment of the same voltage level in the whole process should be disconnected for the test method for AC voltage withstand test of GIS existing now. Because the voltage of the device in the GIS AC voltage 
withstand test system and the voltage in the bus bar are two relatively independent systems, the voltage frequency and voltage phase of them are not always in the same situation. In the interval of the GIS device being expanded or repaired, whether the use of series resonance method to test or the use of reactor for parallel compensation of the wiring method to test, the absolute consistency of AC voltage test voltage and bus operating voltage in the amplitude can not be guaranteed.The expansion and maintenance part of the operation of the GIS equipment and the original operation part of the switch only being isolated and disconnected by the running bus switch. There is a closely relationship among the voltage at fracture, voltage in test system and the voltage on the bus bar . The voltage value of the bus isolation switch is determined by the difference between the frequency and the phase of the test system voltage and the running bus voltage. So the voltage of the isolated switch in the test process is uncertain in time. In this case, the charged GIS AC withstand voltage test is likely to lead to a breakdown of the isolated fracture surface.

In order to avoid the occurrence and occurrence of this phenomenon, we must carry on the same frequency and phase AC voltage test of GIS with no power failure. First of all, the technical personnel of the operation test must clear analysis the change of the voltage on the bus isolation. Take voltage variation in the charged AC voltage test of the same frequency and phase after the expansion or maintenance of the GIS equipment for example, assuming the running bus I and bus II of the same frequency and phase GIS AC voltage test were charged operation in the same voltage grade GIS equipment without power outages.

According to the experimental design and procedure of the same frequency and phase GIS AC voltage withstand test, break off D1,D2 and D3, which are used as a ground knife gate, and close the isolated switch DS1 and circuit breaker CB1 at the same time. At this time, the total voltage bore by switch fracture DS2 and DS3 under the state of isolation is the amplitude difference between the test voltage and the operating voltage.There are mainly three different situations in calculating the voltage difference in the process of pressure test.

\section{Computational method for both frequency and phase between test voltage and operating voltage are different}

When the voltage of GIS same frequency and phase AC voltage test and the voltage of the running bus are different because of the difference in the frequency and phase, The specific formula for calculating the voltage value of the isolated switch is as follow:

$$
\Delta \mathrm{U}=\mathrm{U}_{1}-\mathrm{U}_{2}=\sqrt{2} \mathrm{u}_{1} \sin \left(\omega_{1} \mathrm{t}-\varphi_{1}\right)-\sqrt{2} \mathrm{u}_{2} \sin \left(\omega_{2} \mathrm{t}-\varphi_{2}\right)
$$

Normally,the test frequency of the same frequency and phase AC voltage test of GIS is maintained between $30 \mathrm{~Hz}$ and $100 \mathrm{~Hz}$, or $30 \mathrm{~Hz}<\mathrm{f} 1<100 \mathrm{~Hz}$. Then there must be a numerical $\mathrm{t}$ which is able to simultaneously satisfy the two way requirements of $\mathrm{U} 1$ takes the maximum value and $\mathrm{U} 2$

takes the minimum value, $\Delta U_{\max }=\sqrt{2}(\mathrm{u} 1+\mathrm{u} 2)$. The actual voltage bore by the isolated switch of the same frequency and phase AC voltage test of GIS is the most extreme condition of the circuit. That the test voltage and the operating voltage showing the opposite trend in the phase. And when the amplitude difference between them achieves the maximum peak, the phase difference would be one hundred and eighty degrees. In this extreme case, voltage waveform of Isolated switch of the same frequency and phase GIS AC voltage withstand test is as shown in Figure 1.

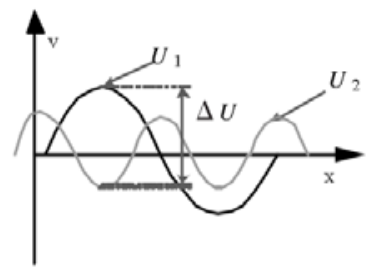

Figure 1 Schematic diagram of isolated switching voltage in extreme cases

From the waveform diagram of Figure 1, it can be seen that the voltage on the isolated switch is the sum of the amplitude of the AC voltage test voltage and the amplitude of the bus running voltage. When the voltage on the isolating switch fracture reaches the maximum value, the voltage 
of isolated switch would endanger the GIS operating equipment in the test because of the burst breakdown phenomenon.

\section{Computational method for the same frequency and different phase between test voltage and operating voltage}

When the frequency between the voltage of GIS same frequency and phase AC voltage test and the voltage of the running bus is different and the phase is equal, which means $\omega_{1=} \omega_{2=} \omega_{0}$ and $\varphi_{1} \neq \varphi_{2}$, the specific formula for calculating the voltage value of the isolated switch is as follow:

$$
\Delta \mathrm{U}=\mathrm{U}_{1}-\mathrm{U}_{2}=\sqrt{2} \mathrm{u}_{1} \sin \left(\omega_{0} \mathrm{t}-\varphi_{1}\right)-\sqrt{2} \mathrm{u}_{2} \sin \left(\omega_{0} \mathrm{t}-\varphi_{2}\right)
$$

Only when U meeting $\left|\varphi_{1}-\varphi_{2}\right|=\pi$, Maximum Umax= (u1+u2) can be taken. So in this case, there still exist the risk of sudden breakdown phenomenon because of that the voltage value of the isolation switch is the maximum.

Computational method for the same frequency and phase between test voltage and operating voltage

If the frequency and phase of the test voltage and the operating voltage are all the same, which means $\omega_{1=} \omega_{2}=\omega_{0}$ and $\varphi_{1}=\varphi_{2}$, the specific formula for calculating the voltage value of the isolated switch is as follow:

$$
\Delta \mathrm{U}=\mathrm{U}_{1}-\mathrm{U}_{2}=\sqrt{2} \mathrm{u}_{1} \sin \left(\omega_{1} \mathrm{t}-\varphi_{1}\right)-\sqrt{2} \mathrm{u}_{2} \sin \left(\omega_{2} \mathrm{t}-\varphi_{2}\right)=\sqrt{2} \sin \left(\omega_{0} \mathrm{t}\right)\left(\mathrm{u}_{1}-\mathrm{u}_{2}\right)
$$

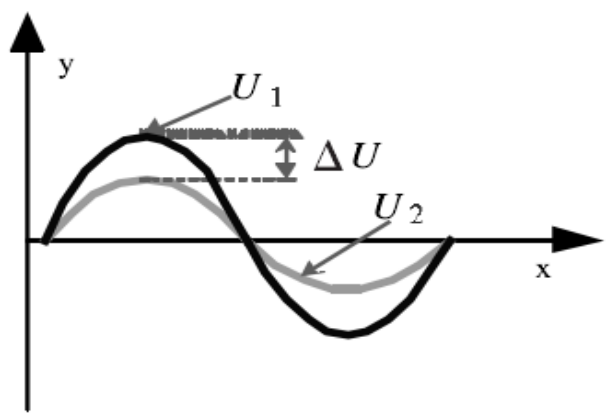

Figure 2 Schematic diagram of voltage waveform of isolating switch in the same frequency and phase

From Figure 2 it is easy to be seen that the voltage value of isolated switch in the same frequency and phase AC voltage test of GIS is the D-value between the amplitude of test voltage and the maximum value of operating voltage. Because the voltage value is very low, there wouldn't be the phenomenon and situation that the fracture being burst breakdown and endanger GIS operating equipment.

Through above analysis of voltage variation In the same frequency and phase AC voltage test of GIS under three different conditions, two conclusions can be summarized. First, when do the AC withstand voltage test using GIS equipment under the premise of no power outage, be sure to pay close attention to the consistency and difference in frequency and phase between test voltage and bus running voltage. Once found a difference between the two, immediately to check whether there is an abnormal circuit between the test voltage and the operating voltage. Otherwise, the voltage at the break of the isolated switch would be at maximum because of that the test voltage is superimposed on the reverse operation voltage. And then there will be a danger of causing the sudden breakdown situation. Second, the voltage that bore by the isolated switch fracture would be stable and safety only if the frequency and phase of the AC withstand test voltage and the bus operating voltage are highly consistent. And there would not be any negative impact or damage to the operation of the original GIS equipment and the interval under expansion or maintenance. 


\section{Conclusion}

The test method of the same frequency and phase AC voltage of GIS has the characteristics of practicality, safety and stability.Compared with the conventional GIS method, the range and the range of the test is more extensive.Improvement and innovation of the method is conducive to enhance the reliability of GIS substation and the harmonious development of economy and society.

\section{Reference}

[1] Wu Gaolin, Xu Ruilin,etc.Simulation analysis and prevention measures of the bus bar insulation breakdown in the AC withstand voltage test of GIS equipment on power J. High Voltage Apparatus, 2013,04:98-104.

[2] Zhao Jinming, Duan Xiao Hua. Application of variable frequency series resonance technology in high voltage cable transfer test J. Power Grid and Clean Energy, 2012,04:23-25.

[3] $\mathrm{Wu}$ wei,application study of GIS equipment of the same frequency and phase ac withstand voltage test technology [J].Science innovation and application, 2014, 4:164-165.

[4] Yin Hua,Wang Qian,Long Yingkai.Questions in GIS equipment of the same frequency and phase ac withstand voltage test technology J.Smart grid, 2015,07:679-682.

[5] Wu Gaolin,Xu Ruilin,Xiao Qianbo.Simulation analysis and prevention measures of the bus bar insulation breakdown in the AC withstand voltage test of GIS equipment J.High voltage apparatus, 2013,04:98-104.

[6] Li Zhongsheng. Calculation of the set of the reactor in series resonant voltage withstand test J. Journal of Hunan Industry Polytechnic, 2014,01:4-6. 